\title{
Predicting the formation and stability of single phase high-entropy alloys
}

\author{
Authors: D.J. M. King ${ }^{\mathrm{a}, \mathrm{b}^{*}}$, S.C. Middleburgh ${ }^{\mathrm{c}}$, A.G. McGregor ${ }^{\mathrm{d}}$, M.B. Cortie ${ }^{\mathrm{b}}$
}

\author{
Affiliations: \\ ${ }^{a}$ Institute of Materials Engineering, Australian Nuclear Science and Technology \\ Organisation, Lucas Heights, NSW, Australia. \\ ${ }^{\mathrm{b}}$ Institute for Nanoscale Technology, University of Technology Sydney, NSW, Australia. \\ ${ }^{c}$ Westinghouse Electric Sweden AB, SE-721 63 Västerås, Sweden \\ 'Tyro Payments, 155 Clarence St, Sydney, NSW, Australia. \\ *daniel.miks@live.com
}

Abstract: A method for rapidly predicting the formation and stability of undiscovered single phase high-entropy alloys (SPHEAs) is provided. Our software implementation of the algorithm uses data for 73 metallic elements and rapidly combines them - 4, 5 or 6 elements at a time - using the Miedema semi-empirical methodology to yield estimates of formation enthalpy. Approximately 186,000,000 compositions of 4, 5 and 6 element alloys were screened, and $\sim 1,900$ new equimolar SPHEAs predicted. Of the 185 experimentally reported HEA systems currently known, the model correctly predicted the stability of the SPHEA structure in 177 . The other sixteen were suggested to actually form a partially ordered solid solution - a finding supported by other recent experimental and theoretical work. The stability of each alloy at a specific temperature can also be predicted, allowing precipitation temperatures (and the likely precipitate) to be forecast. This combinatorial algorithm is described in detail, and its software implementation is freely accessible through a webservice allowing rapid advances in the design, development and discovery of new technologically important alloys. 
Keywords : Computational thermodynamics; High-entropy alloys; Density functional theory; Thermodynamic stability; Order-disorder phenomena;

\section{Introduction}

Exploratory alloy discovery, design and development is a crucial aspect of materials research for the growth of industries such as aerospace [1], biomedical [2, 3] and energy generation and storage [4]. Recently, this aspect of material research has expanded past that of the conventional alloy (consisting of 1 or 2 principle components) into a new class of highly alloyed materials, high-entropy alloys (HEAs). This field has gained attention [5-7] due to findings of higher strengths $[8,9]$, ductility [10] and other interesting properties [11] pertaining to this class. Efforts to design, characterise and utilise HEAs are currently underway globally. However, the meaning of the term 'HEA' is not continuous across the literature. Taken as written, the HEA definition provided by Yeh et al [7], and re-iterated by others since then $[1,12]$, defines a HEA as any alloy consisting of 5 or more elements between $5-35$ at. \%. As such, this type of HEA will usually possess a microstructure consisting of two or more distinct phases, some of which are likely to be brittle intermetallic compounds. Although, multiphase strengthening is sometimes desirable in alloys, a large amount of any brittle phase will generally make an alloy unusable as a structural material and such HEAs are therefore mundane. However, as will be discussed shortly, in a few special cases, the additional entropy of the multi-element composition will stabilise a microstructure consisting of either (i) a single solid solution having one of the simple close-packed crystal structures (FCC, HCP or BCC) or (ii) a duplex microstructure consisting of two such simple solid solutions. Generally, it is these non-trivial examples of HEA that interest investigators. This is because the resulting random solid solution(s) will exhibit a combination of ductility coupled with significant solid solution hardening. We recommend use of the term single 
phase high-entropy alloy (SPHEA) as a more restrictive term to differentiate the rare and desirable single phase type of HEA from generic examples of multiphase HEAs.

Combining Yeh's compositional limits [7] with the requirement for a single phase solid solution, we can define a SPHEA as an alloy with $\geq 4$ alloying elements, at least 4 of which with a molar ratio between $0.33-1$ to that of the highest contributing element. These alloys must be able to display a single phase of simple, random, close-packed structure below the solidus.

We return now to the factors that might stabilise a SPHEA. The prevailing hypotheses were (i) that the simple crystal structure or structures are thermodynamically stabilised, relative to possible intermetallic compounds, by the increased configurational entropy from large alloying additions, (ii) that the absence of chemical ordering and the presence of the simple crystal structure should provide dislocation mobility and hence ductility similar to the known structural alloys, such as steels, and (iii) higher strengths can be achieved due to the large concentrations of the elements within the solid-solution which contributes to an intrinsic lattice strain, and the so-called "cocktail effect" [13].

Some complications exist that place constraints on the utility of these hypotheses. For example, while configurational entropy of any solid solution would certainly increase as more elements are added to a composition, there is also an increased likelihood that the introduction of an additional alloying element will cause the formation of a stable new intermetallic phase. This concept is hypothesised by Zhang et al. [14] and later confirmed by Senkov et al. [15]. Nevertheless, the number of possible combinations is exceedingly large and it is considered feasible that many useful SPHEAs exist. 
The basic assumption underlying the formation of SPHEAs can be explained by considering the equation for Gibbs free energy:

$\Delta G=\Delta H-T \times \Delta S$

It follows that $\Delta G$ can be rendered negative (i.e. the new phase can be stabilised) by a sufficiently large entropy, $\Delta S$, which is contributed to by the configurational entropy, $\Delta S_{\text {conf }}$. Importantly, this stabilisation can occur even if the enthalpy of mixing, $\Delta H$, is positive. $\Delta S_{\text {conf }}$ is related to the number of ways that the material's atoms can be arranged $(\omega)$ as:

$$
\Delta S_{\text {conf }}=k_{B} \ln \omega=-n R \sum_{i}^{j} x_{i} \ln \left(x_{i}\right)
$$

where $k_{B}$ is Boltzmann's constant, $n$ is the number of moles, $R$ is the gas constant, $x$ is the atomic fraction of element $i$ in a $j$ element system.

It was first postulated by Yeh et al. [7] that, in alloys consisting of five or more elements in near equimolar concentrations, the formation of intermetallic compounds could be suppressed by the alloys' increased $\Delta S_{\text {conf. }}$. However, over the last decade or so it has become apparent that this prediction often fails [16-18]. It is evident that the existing predictive framework is incomplete and that the theory must be adjusted to correctly describe these observations. Indeed, many parameters [19] have been proposed as a means of predicting the formation of novel SPHEAs, yet none have been able to reliably separate intermetallic phases from solid solution in the absence of existing experimental or modelled thermodynamic data. Where such data is available, the CALPHAD method has been applied to this problem and, for example, was recently used to screen $~ 130,000$ compositions [15]. However, even in the CALPHAD approach incorrect predictions occur. Furthermore, the sheer number of alloy systems encountered when exploring beyond ternary systems makes the calculation of the Gibbs free energy of unexplored solid solutions both costly experimentally [20] and 
theoretically $[21,22]$. In addition, a simplifying scheme to represent the stability of such systems on a 2D diagram (akin to phase diagrams that can represent up to three variables) does not exist.

Here we apply Miedema's macroscopic atom model [23], for the calculation of concentrated solid solutions, to this problem. The existing model is extended to account for multicomponent systems and implemented within a new framework to utilise 73 elements of the periodic table. This technique is implemented here in software [24]. The 185 experimentally studied alloys described in past literature are used to benchmark the new algorithm. Furthermore, we present a new way to represent these multicomponent systems on a 2D medium that allows for quick analysis of the systems stability. Fifty of the lowest cost, fourelement, equimolar systems are reported.

\subsection{Current state-of-the-art}

In Yeh et al.'s original publication [7] it is stated that the configurational entropy of an equimolar alloy consisting of five elements is of the same magnitude as the configurational entropy of a strong intermetallic compound. However, in an ordered system the configurational entropy equates to zero, from Eq. (2). Although other contributions to entropy such as vibrational, electronic and magnetic moment randomness can be present, these entropic contributions are often similar in ordered, segregated and solution phases of the system $[25,26]$, and therefore cancel in comparison. It is mostly the non-zero configurational entropy of a disordered solution system that is thought to stabilise the solid solution phase. Nevertheless, one must consider all aspects of the Gibbs free energy equation in order to draw conclusions about the nature of system stability. 
At the heart of current HEA phase prediction models [27], $\Delta H$ of a HEA is defined as:

$$
\Delta H=\sum_{\substack{i=1 \\ i \neq j}}^{n} 4 \Delta H_{i j}^{a m} c_{i} c_{j}
$$

where $c$ is the concentration, in atomic fraction, of element $i$ and $j$, and $\Delta H_{i j}^{a m}$ is the enthalpy of mixing per mole between element $i$ and $j$ calculated by the equation for a liquid binary alloy in Miedema's model [28]. There are some associated errors with this model [29] but more concerning is that it only represents an ideal solution and assumes a 50/50 mixture will yield the largest magnitude in the enthalpy of mixing [30]. Nevertheless, a ratio of the two contributions to the Gibbs free energy was proposed by Yang et al. [31]:

$\Omega=\frac{T_{m} \Delta S_{\text {conf }}}{|\Delta H|}$

A disordered solid solution is expected to form for $\Omega \geq 1$.1. However, this comparison does not include any information of the solid solution phase nor intermetallic compounds. This is because neither the enthalpy of formation of the solid solution $\left(\Delta H_{s s}\right)$ or of the intermetallic $\left(\Delta H_{\text {int }}\right)$ is included, and instead, it is thought that $\Delta H_{a m}$ is indicative of $\Delta H_{s s}$ and $\Delta H_{\text {int }}$. In fact, the maximum absolute difference in $\Delta H_{a m}-\Delta H_{s s}$, and $\Delta H_{a m}-\Delta H_{\text {int }}$ (for the 185 systems benchmarked in this work) is $19 \mathrm{~kJ} / \mathrm{mol}$ and $67 \mathrm{~kJ} / \mathrm{mol}$, respectively, which would suggest errors of this magnitude should be expected when using this method. In Yang et al.'s original paper [27] it is shown that HEAs which display intermetallic compounds cannot be discerned from those that do not. This effect is replicated in Figure 1. 
The well-known Hume-Rothery rules take the size of the alloy's constituent atoms into account. The HEA community generally expresses this factor as a dimensionless representation of the difference in atomic radii, indicated as an indirect representation of the strain within the material [19],

$$
\delta=\sqrt{\sum_{i=1}^{n} c_{i}\left(1-r_{i} /{ }^{r}\right)^{2}} \times 100
$$

where $r$ is commonly taken to be the Goldschmit atomic radius of element $i$ and $\bar{r}$ is the weighted average of the systems atomic radii. This is also included in Yang et al.'s prediction whereby a value of $\delta \leq 6.6$ indicates a disordered solid solution should form [27].

Two purely geometrical parameters $\Lambda$ and $\gamma$ have recently been developed [19, 32], however their predictive capability is not much different from $\Omega$ and $\delta$, see Supplementary Material, Figure S1.

\section{Methodology}

\subsection{Empirical calculations}

A new method for predicting the formation of a HEA is employed within this work. Two values are compared to assess the suppression of an ordered phase through the formation of a random solid solution:

1. $\Delta G_{s s}$, the change in Gibbs free energy for the formation of a fully disordered solid solution from a mixture of its individual elements.

2. $\Delta G_{\max }$, the lowest (intermetallic) or highest (segregated) possible Gibbs free energy obtainable from the formation of binary systems from the constituents of the mixture. 
The values for the Gibbs free energy are calculated using Eq. (1) whereby the values for the enthalpy of mixing of the solid solution, $\Delta H_{s s}$, and intermetallic, $\Delta H_{\text {int }}$, are calculated using Eq. (S.8) and (S.9), respectively, the temperature is taken as the melting temperature from the rule of mixtures and $\Delta S_{\text {conf }}$ from Eq. (2). The contribution of other sources of entropy to $\Delta S$ is assumed to be very similar and therefore cancel between $\Delta G_{s s}$ and $\Delta G_{\text {max }}$. Since $\Delta S_{\text {conf }}$ is 0 for ordered intermetallics, we assume the $\Delta G_{\text {int }}=\Delta H_{\text {int }}$.

The binary system that yields the largest magnitude of $\Delta G_{\max }$ is determined by ranking the magnitude of $\Delta G_{\text {int }}$ of all the possible binaries obtainable by the system. This value is then scaled to maintain stoichiometry of the system.

These values are combined to form a new parameter:

$\Phi=\frac{\Delta G_{s s}}{-\left|\Delta G_{\max }\right|}$

Where a value of $\Phi \geq 1$ would suggest a stable solid solution at the systems' melting temperature and negative value of $\Phi$ would suggest the solid solution has positive formation enthalpy and will not form.

\subsection{First principles}

A plane wave density functional theory (DFT) package, VASP [33, 34], was used to calculate the ground state energies of the ordered and disordered AlCoFeNi HEA, the CoFe and AlNi binary intermetallic compounds, alloys in the Mo-Nb-Ti-V system, and a range of compounds in the Pt-Al system. The plane wave code was chosen as it is known to reproduce the intermetallic/metallic nature of the bonding to a satisfactory accuracy. 
AlCoFeNi and the Mo-Nb-Ti-V system were chosen as prototypical examples of HEA systems, while the Pt-Al system was chosen to benchmark the accuracy of the techniques used to calculate enthalpies.

FCC Al, Co, Fe and $\mathrm{Ni}$, and $\mathrm{BCC} \mathrm{Cr}$ were converged independently. A $k$-point density of $\sim 0.035 \AA^{-1}$ was found to satisfy the lattice energy convergence criteria of $\Delta \mathrm{E}_{\text {lat }} \leq 1 \times 10^{-3} \mathrm{eV}$. Similarly BCC Mo, Nb, V, and HCP Ti were converged to obtain a $k$-point density of $\sim 0.026$ $\AA^{-1}$ and FCC Pt and FCC Al: $\sim 0.023 \AA^{-1}$. These respective $k$-point densities were maintained for all calculations for the respective systems. A Methfessel-Paxton smearing method $(0.125$ $\mathrm{eV})$ was used and the maximum number of valence electrons were treated as valence states for all elements. This was achieved by using the supplied pseudo-potentials within the VASP package. These computations were performed under constant pressure using the GGA-PBE exchange correlation functional, and accounting for spin-polarisation effects (including magnetism). The energy convergence criteria for all calculations were within $10^{-4} \mathrm{eV}$ per supercell. The methods for the modelling the BCC and FCC disordered HEAs were described elsewhere $[22,35]$. Thirty points on the Mo-Nb-Ti- $\mathrm{V}_{0.25}$ ternary phase diagram were systematically chosen for a broad coverage and their formation enthalpies were calculated. The formation enthalpies for $\mathrm{Al}_{2} \mathrm{Pt}, \mathrm{Al}_{3} \mathrm{Pt}, \mathrm{Al}_{4} \mathrm{Pt}, \mathrm{Al}_{5} \mathrm{Pt}$ and $\mathrm{Al}_{6} \mathrm{Pt}$ compounds were calculated according to the calculation:

$$
\Delta H=\frac{1}{x+y} E\left(\mathrm{Al}_{x} \mathrm{Pt}_{y}\right)-\left(\frac{y}{x+y} E(\mathrm{Pt})+\frac{x}{x+y} E(\mathrm{Al})\right)
$$

where $E\left(\mathrm{Al}_{x} \mathrm{Pt}_{y}\right)$ is the energy of a supercell simulating the intermetallic compound. The terms $E(\mathrm{Pt})$ and $E(\mathrm{Al})$ represent the lattice energies of a single atom from pure FCC $\mathrm{Pt}$ and Al respectively. The formation enthalpies of all other alloys were calculated using a similar equation. 


\subsection{Analysis of experiments}

Overall, 185 alloy systems, previously experimentally analysed using structural and elemental analysis, were used to benchmark the $\Phi$ value, (see Supplementary Material, Table S1). We define the HEAs, within the literature, into three categories.

1. Solid solutions: Systems that display reflections, in diffraction data, analogous to BCC, FCC or HCP and are devoid of intermetallics. These are single-phase materials although they might display some chemical segregation due to coring during dendritic solidification.

2. Intermetallic/multiphase: Systems that display reflections, in diffraction data, of an intermetallic compound or of a second solid solution or element.

3. Exceptions: Systems that display reflections, in diffraction data, analogous to BCC, FCC or HCP and are devoid of intermetallic phases but are predicted by the current method to form an intermetallic/multiphase structure.

\section{Results}

\subsection{Benchmarking to experimental literature}

We start by comparing the results of Miedema's empirical approach to that of experimental and quantum mechanical enthalpies. Although the quantum mechanical basis for Miedema's model is tenuous at best [36], past studies have shown that the values obtained show a very similar trend when compared to experiment [37] and ab intio techniques [38]. As mentioned, we selected the Pt-Al and Mo-Nb-Ti- $\mathrm{V}_{0.25}$ systems to make further comparisons. Pt-Al was chosen as it presents a large range of intermetallic compounds all of which having a wealth of 
experimental and theoretical data. Mo-Nb-Ti- $\mathrm{V}_{0.25}$ was chosen as it represents a system that is devoid of intermetallic compounds, save for a metastable $\mathrm{NbTi}_{4}$ phase [39].

The formation enthalpies of 10 binary intermetallic compounds within the Pt-Al system were calculated using Miedema's model and DFT, see Figure 2. There is a clear underestimation of the magnitude when compared to the convex hull, with a difference as large as $\sim 20 \mathrm{~kJ} / \mathrm{mol}$ for $\mathrm{Al}$ contents of 50 at. \%. This effect is also seen in the comparison of the formation enthalpy of the solid solution for Mo-Nb-Ti- $\mathrm{V}_{0.25}$, see Figure 3. Importantly, however, the trends between the datasets remain the same. Although the magnitudes of values are not precise, the relative values are very similar and therefore can be compared within the same model.

Next, we apply our methodology to the question of SPHEAs by exploring three different systems: An alloy that, (i) forms a single phase solid solution - CoCrFeNi [40], (ii) has a segregation of 1 element - CoCrCuFeNi [41, 42], and (iii) forms an intermetallic compound $\mathrm{AlCoCrFeNi}[43,44]$.

i) Single phase solid solution - $\mathrm{CoCrFeNi}$ :

$\mathrm{Co}+\mathrm{Cr}+\mathrm{Fe}+\mathrm{Ni} \rightarrow(\mathrm{CoCrFeNi})_{\mathrm{ss}}$ $\Delta G_{s s}=-23.43 \mathrm{~kJ} / \mathrm{mol}$ (from Eq. (1))

$\mathrm{Cr}+\mathrm{Ni} \rightarrow \mathrm{CrNi}$

$\Delta G_{\text {int }}=-10.08 \mathrm{~kJ} / \mathrm{mol}$ (from Eq. (S.9))

Since $\Delta G_{\text {int }}$ of $\mathrm{CrNi}$ is of the largest magnitude of the possible intermetallics, we extend this value to include all 4 elements. This implies the formation of CoFe is also of the same 
magnitude and that the ordered result is of the largest possible magnitude (overestimating the drive for segregation).

ie. $\mathrm{Co}+\mathrm{Cr}+\mathrm{Fe}+\mathrm{Ni} \rightarrow \mathrm{CrNi}+\mathrm{CoFe}$

$$
\Delta G_{\max }=-20.16 \mathrm{~kJ} / \mathrm{mol}
$$

Following, $\Phi=1.16$ (Eq. (6)) predicting a single phase solid solution is thermodynamically stable at the system's melting temperature.

ii) Segregated - CoCrCuFeNi:

$\mathrm{Co}+\mathrm{Cr}+\mathrm{Cu}+\mathrm{Fe}+\mathrm{Ni} \rightarrow(\mathrm{CoCrCuFeNi})_{\mathrm{ss}}$

$$
\Delta G_{s s}=-21.08 \mathrm{~kJ} / \mathrm{mol}
$$

$\mathrm{Cu}+\mathrm{Fe} \rightarrow \mathrm{CuFe}$

$$
\Delta G_{\text {int }}=+19.14 \mathrm{~kJ} / \mathrm{mol}
$$

$\mathrm{Co}+\mathrm{Cr}+\mathrm{Cu}+\mathrm{Fe}+\mathrm{Ni} \rightarrow \mathrm{CuFe}+\mathrm{MM}+\mathrm{M}$

$$
\Delta G_{\max }=+38.28 \mathrm{~kJ} / \mathrm{mol}
$$

Where, $\mathrm{M}$ is used to denote an arbitrary allocation of the remaining elements.

In this case, $\Phi=0.55$ predicts that a single phase solid solution is not thermodynamically stable.

iii) Intermetallic - $\mathrm{AlCoCrFeNi}$

$\mathrm{Al}+\mathrm{Co}+\mathrm{Cr}+\mathrm{Fe}+\mathrm{Ni} \rightarrow(\mathrm{AlCoCrFeNi})_{\mathrm{ss}}$

$$
\Delta G_{s s}=-33.89 \mathrm{~kJ} / \mathrm{mol}
$$




$$
\Delta G_{\text {int }}=-47.33 \mathrm{~kJ} / \mathrm{mol}
$$

$\mathrm{Al}+\mathrm{Co}+\mathrm{Cr}+\mathrm{Fe}+\mathrm{Ni} \rightarrow \mathrm{AlNi}+\mathrm{MM}+\mathrm{M}$

$$
\Delta G_{\max }=-94.66 \mathrm{~kJ} / \mathrm{mol}
$$

Equating to, $\Phi=0.36$, also predicting a single phase solid solution is not stable.

Figure 4 shows a clear distinction between the HEAs reported to display a disordered solution (blue) to those that were reported to display intermetallic/multiphase phases (red), at $\Phi=1$ (dashed line), with the exception of 16 systems (green) that were reported to be single phase solid solutions.

The $\delta$ value (Eq. (5)) from the Hume-Rothery rules, is again employed as previous results by Yang et al. [27] suggests that no SPHEA exists for $\delta \geq 6.6$. Interestingly, the $\mathrm{NbTiV}_{2} \mathrm{Zr}$ alloy is the only system that has both $\Phi>1$ and $\delta>6.6$. Senkov et al. [45] reported a tri-phase $\mathrm{BCC}$ microstructure with segregation between $\mathrm{V}$ and $\mathrm{Zr}$ into phases with the compositions $\mathrm{Nb}_{0.2} \mathrm{Ti}_{0.2} \mathrm{~V}_{0.1} \mathrm{Zr}_{0.5}, \mathrm{Nb}_{0.2} \mathrm{Ti}_{0.2} \mathrm{~V}_{0.5} \mathrm{Zr}_{0.1}$ and $\mathrm{Nb}_{0.2} \mathrm{Ti}_{0.2} \mathrm{~V}_{0.4} \mathrm{Zr}_{0.1}$. A thermodynamic analysis reveals the $\mathrm{V}_{2} \mathrm{Zr}$ C15 Laves phase is the most likely candidate for compound formation which, itself, has a positive formation enthalpy [46]. Therefore, it is plausible that a segregated microstructure is preferred. This aligns with the findings of Senkov et al., where it was found that homogenisation of this alloy does not occur after annealing [45].

Figure 5 highlights the relationship between the enthalpy of mixing of the intermetallic with the largest magnitude, $\Delta H_{\max }$, and alloy prediction. For $\Delta H_{\max }<0 \mathrm{~kJ} / \mathrm{mol}$, if a binary intermetallic compound is stable enough $(\leq 17.5 \mathrm{~kJ} / \mathrm{mol})$, the complete solid solution is destabilised. When $\Delta H_{\max }>0 \mathrm{~kJ} / \mathrm{mol}$ a similar effect is observed. However, a positive Gibbs free energy denotes an unstable reaction for the formation of the binary intermetallic. 
Therefore, the physical premise of this observed relationship is that the chemical interaction between the two elements in the hypothetical binary system is sufficiently unfavourable, that within the solid solution, this interaction will also act to cause instability.

Due to the rigorousness of this method, it is possible that some SPHEAs exist for $\Phi<1$. The inclusion of enthalpies of formation of the less favourable intermetallics, ternary intermetallics, minor alloying/segregation and multiple solid solutions will act to lower the magnitude of the $\Delta G_{\max }$ value. However, to include these factors does not allow for a quick and simple calculation to be implemented into a single parameter. We therefore proceed with the current method as a first approximation for SPHEA formation.

\subsection{Predicting precipitation temperatures}

It is possible to estimate the temperature at which the solid solution will destabilise by calculating the temperature at which the alloy is predicted to have a value of $\Phi=1$ (instead of calculating at the alloy's predicted melting temperature). These results were found to be in agreement with past experimental results, especially for systems with higher transition temperatures. For example, this method predicts that NbTaTiHfZr, NbHfTiZr and MoNbTaVW are stable above $1162 \mathrm{~K}, 1359 \mathrm{~K}$ and $1580 \mathrm{~K}$, respectively, which aligns well with the results by Senkov et al. [47], Wu et al. [48] and Zou et al [49] who studied the homogenisation and structural stability during annealing of these alloys. However, difficulties in accurately identifying the temperature in which precipitates occur at low temperatures arise due to kinetic effects. This is highlighted in a study by Tsai et al. [50] on the CoCrFeMnNi system whereby high activation energies for diffusion within the alloy were measured. 


\subsubsection{Exploring the Co-Cr-Fe-Ni system}

We now explore the Co-Cr-Fe-Ni system in detail. All stoichiometries with a step-size of 1 at. $\%$ are computed and shown in Figure 6.

By considering all of the potential stoichiometries we predict that the $\mathrm{Co}_{0.43} \mathrm{Cr}_{0.01} \mathrm{Fe}_{0.07} \mathrm{Ni}_{0.49}$ composition is the most stable stoichiometry of this system displaying a value of $\Phi=8.93$. This prediction arises as $\mathrm{Cr}-\mathrm{Fe}$ forms a strong $\sigma$ phase intermetallic in their binary phase diagram [14] but complete segregation of these species will lead to a less favourable Gibbs free energy of the solid solution phase. The balance of this interplay results in a Co and $\mathrm{Ni}$ rich alloy. The equimolar alloy is comparatively less compositionally stable with a value of $\Phi$ $=1.16$. To benchmark these results, the segregation of $\mathrm{Co}, \mathrm{Cr}, \mathrm{Fe}$, and $\mathrm{Ni}$, from the equimolar system is tracked, see Figure 6. This method predicts that this system favours the segregation of $\mathrm{Cr}$ from the equimolar system as the temperature is reduced and the segregation of all other species has a relatively negligible effect. This is evident as $\Phi$ increases as $\mathrm{Cr}$ content is decreased, a behaviour supported by both experimental [51] and computational work [22]. This model also predicts that the removal of Ni will also contribute to a slightly higher stability compared to the equimolar alloy, but less $\mathrm{Cr}$ is required to segregate to achieve a higher stability. The precipitation temperature of $\mathrm{Cr}$ is estimated to be $1156 \mathrm{~K}$ when $\mathrm{Cr}$ is at 11.8 at. \%. Due to kinetic hindrance at lower temperatures, prolonged annealing times [52] may be required to observe further $\mathrm{Cr}$ segregation.

\subsection{Exceptions to the model}

Of the 185 alloys used to benchmark this new method, 16 exceptions were found. All of these exceptions include $\mathrm{Al}$ within their mixtures and also display $H_{\max }$ values of the same 
magnitude as the intermetallic/multiphase systems screened (Figure 5). It has been reported that HEAs with $\mathrm{Al}$ can exist in the form of partially ordered $\mathrm{BCC}$ alloys, the nature of which might not be evident through standard experimental analysis $[53,54]$. We now investigate this drive for ordering, on the atomic scale, for one of the exceptions, AlCoFeNi, using DFT.

For a fully disordered AlCoFeNi BCC HEA

$\Delta H_{d i s}=-22.79 \mathrm{~kJ} / \mathrm{mol}$

In contrast, partial ordering in which $\mathrm{Al}$ and $\mathrm{Ni}$ are restricted to the centre and corner site of the BCC lattice, respectively, and Co and Fe are allowed to occupy either, has a significantly more negative enthalpy of mixing:

$\Delta H_{\text {ord }}=-28.85 \mathrm{~kJ} / \mathrm{mol}$

The configurational entropy of the partially ordered system was calculated using a method previously reported [54]. The partially ordered configurational entropy was calculated to be $2.88 \times 10^{-3} \mathrm{~kJ} / \mathrm{K} / \mathrm{mol}$ less than that of the fully disordered system. Figure 7 shows the Gibbs free energy as a function of temperature for these two configurations, accounting for the difference in $\Delta S_{\text {conf }}$, with a comparison to a dual phase $\mathrm{AlNi}+\mathrm{CoFe}$ intermetallic formation.

Here it is clear that the AlCoFeNi system favours a partially ordered crystal structure compared to the fully disordered alternative. Under the assumption that this system does not favour a different ordering, it is predicted that the partially ordered system with BCC packing will be adopted under equilibrium conditions at temperatures $\geq 1220 \mathrm{~K}$. These findings may provide some insight into the high hardness and yield strength reported by Zuo et al. [55] for this material. It is possible that this relationship exists in the other $15 \mathrm{Al}$-containing exceptions as well. However, it should be noted that in this specific example we consider the enthalpy of mixing of $\mathrm{CoFe}(-13.93 \mathrm{~kJ} / \mathrm{mol})$ which is considerably more positive than AlNi. 
Certainly when scaling the magnitude of CoFe to that of AlNi (as is done in the current $\Phi$ evaluation) the notion of partial ordering is irrelevant and a prediction of intermetallic/multiphase is made. It is evident that Miedema's model cannot precisely identify that these systems will form a single phase solid solution (partially ordered or otherwise). Therefore it is more likely that the current method is too rigorous and may omit Al-containing alloys from the solid solution category. It is also possible that nanoprecipitates were overlooked within the experiments themselves. In the $\mathrm{Al}_{0.3} \mathrm{CoCrFeNi}$ HEA Shun et al. [56] observed homogeneous nanoprecipitates [8] in the as-cast condition using TEM. After aging at $1173 \mathrm{~K}$ for 72 hours, a B2 ordering was reported which is in excellent agreement with the DFT predictions of this similar system.

\subsection{Predicting new high-entropy alloy systems}

Having established that the $\Phi$ value gives a good first approximation to SPHEA stability and formation, we next searched through all permutations of a mixture of 73 elements from the periodic table, looking for alloys that satisfy $\Phi \geq 1$ and $\delta \leq 6.6$, see Table S2. The resultant structures can then be ranked for properties that scale with the rule-of-mixtures such as bulk modulus, thermal neutron cross-section, melting temperature etc. A total of 186,299,362 equimolar systems were screened (1,088,431 4 element, 15,020,335 5 element, and 170,230,452 6 element systems), 1970 potential SPHEAs were identified (636 4 element, 983 5 element, and 3066 element). We list 50 of the four element systems sorted by estimated price, see Table S2.

We encourage readers to attempt to design their own SPHEA at www.alloyASAP.com. 


\section{Conclusions}

A new method for predicting formation of SPHEAs has been developed, using Miedema's model as a basis. Although the absolute values provided by the Miedema methodology are not rigorously accurate, the trend in alloy systems is essentially the same as that predicted by DFT calculations. We have shown that it is useful to use the Miedema methodology described in the paper to work through very large numbers of alloy compositions, far more permutations - by orders of magnitude - than would be feasible using DFT.

A new parameter, $\Phi$, the ratio between the Gibbs free energy of a completely disordered solid solution to that of the most likely intermetallic or segregated binary system, is defined. This method takes into account both the intermetallic and solid solution enthalpy of mixing in a regular solution model and is validated by applying it to 185 experimentally verified systems from the literature. We show that the formation of HEA random solid solutions is favoured when $\Phi>1$. Of these benchmarked systems, 16 exceptions were found. These were systems reported in the literature as SPHEAs but not identified as such by our methodology. DFT calculations suggest that partial atomic ordering may occur in these discrepancies. While the onset of partial atomic ordering would stabilise the structures more than a model based on a random solid solution would predict, it is unreasonable to conclude that this is the case for all of the exceptions. It is more likely that the current method is too stringent in the calculation of the ordered/multiphase enthalpies of mixing and if more possible phases are calculated there would be a balancing effect to the enthalpy term in which a favourable SPHEA would be predicted. This would explain the omission of Al from any equimolar SPHEA prediction.

Nevertheless, the new stability parameter can be used to predict the temperature at which the single phase solid solution will begin to precipitate. This form of calculation can be used to predict whether compositions will precipitation harden or decompose at a certain 
temperature. Conversely, one can use this method to design an alloy that is not expected to have ageing effects below a given operation temperature. These effects, combined with the scalable material properties are combined in the ASAP code to provide a method to aid alloy design and discovery, optimising for specific material properties.

\section{Acknowledgments:}

This research was undertaken with the assistance of resources provided by the NCI National Facility systems at the Australian National University through the National Computational Merit Allocation Scheme supported by Intersect Australia Ltd and the Australian Government, and the Multi-modal Australian ScienceS Imaging and Visualisation Environment (MASSIVE) (www.massive.org.au). We also thank Prof. Lyndon Edwards, and Drs Greg Lumpkin and Gordon Thorogood (Australian Nuclear Science and Technology Organisation) for their support throughout this project. 


\section{References}

[1] D.B. Miracle, J.D. Miller, O.N. Senkov, C. Woodward, M.D. Uchic, J. Tiley. Exploration and Development of High Entropy Alloys for Structural Applications, Entropy 16 (2014) 494-525.

[2] A. Hynowska, A. Blanquer, E. Pellicer, J. Fornell, S. Suriñach, M. Baró, S. González, E. Ibáñez, L. Barrios, C. Nogués, J. Sort. Novel Ti-Zr-Hf-Fe Nanostructured Alloy for Biomedical Applications, Mater. 6 (2013) 4930-4945.

[3] H. Li, X. Xie, K. Zhao, Y. Wang, Y. Zheng, W. Wang, L. Qin. In vitro and in vivo studies on biodegradable CaMgZnSrYb high-entropy bulk metallic glass, Acta Biomater. 9 (2013) 8561-8573.

[4] I. Kunce, M. Polanski, J. Bystrzycki. Microstructure and hydrogen storage properties of a TiZrNbMoV high entropy alloy synthesized using Laser Engineered Net Shaping (LENS), Int. J. Hydrog. Energy 39 (2014) 9904-9910.

[5] C. Huang, Y. Zhang, R. Vilar, J. Shen. Dry sliding wear behavior of laser clad TiVCrAlSi high entropy alloy coatings on Ti-6Al-4V substrate, Mater. Des. (2012) 338-343.

[6] T. C., M. Tsai, J. Yeh, C. Yang. Effect of temperature on mechanical properties of Al0.5CoCrCuFeNi, J. Alloy. Compd. (2010) 160-165.

[7] J. Yeh, S. Chen, S. Lin, J. Gan, T. Chin, T. Shun, C. Tsau, S. Chang. Nanostructured HighEntropy Alloys with Multiple Principal Elements: Novel Alloy Design Concepts and Outcomes, Adv. Eng. Mater. (2004) 299-303.

[8] Y. Zhang, T.T. Zuo, Z. Tang, M.C. Gao, K.A. Dahmen, P.K. Liaw, Z.P. Lu. Microstructures and properties of high-entropy alloys, Prog. Mater. Sci. 61 (2014) 1-93.

[9] S.C. Lumley, R.W. Grimes, S.T. Murphy, P.A. Burr, A. Chroneos, P.R. Chard-Tuckey, M.R. Wenman. The thermodynamics of hydride precipitation: The importance of entropy, enthalpy and disorder, Acta Mater. 79 (2014) 351-362.

[10] B. Gludovatz, A. Hohenwarter, D. Catoor, E.H. Chang, E.P. George, R.O. Ritchie. A fracture-resistant high-entropy alloy for cryogenic applications, Sci. 345 (2014) 1153-1158.

[11] Y. Zhang, T.T. Zuo, Y.Q. Cheng, P.K. Liaw. High-entropy Alloys with High Saturation Magnetization, Electrical Resistivity, and Malleability, Sci. Rep. 3 (2013) 7.

[12] Y. Zhang, Y.J. Zhou, J.P. Lin, G.L. Chen, P.K. Liaw. Solid-solution phase formation rules for multi-component alloys, Adv. Eng. Mater. 10 (2008) 534-538.

[13] W. Tang, J. Yeh. Effect of aluminium content on plasma-nitrided AlxCoCrCuFeNi highentropy alloy, Metall. Mater. Trans. A (2009) 1479-1486.

[14] F. Zhang, C. Zhang, S.L. Chen, J. Zhu, W.S. Cao, U.R. Kattner. An understanding of high entropy alloys from phase diagram calculations, Calphad - Comput. Coupling Phase Diagr. Thermochem.45 (2014) 1-10.

[15] O.N. Senkov, J.D. Miller, D.B. Miracle, C. Woodward. Accelerated exploration of multiprincipal element alloys with solid solution phases, Nat. Commun. 6 (2015).

[16] Y. Zhou, Y. Zhang, Y. Wang, G. Chen. Microstructure and compressive properties of multicomponent Alx(TiVCrMnFeCoNiCu)100-x high-entropy alloys, Mater. Sci. Eng. A (2007) 260-265.

[17] B. Li, Y. Wang, M. Ren, C. Yang, H. Fu. Effects of Mn, Ti and V on the microstructure and properties of AlCrFeCoNiCu high entropy alloy, Mater. Sci. Eng. A 498 (2008) 482-486. 
[18] Y.-L. Chen, Y.-H. Hu, C.-A. Hsieh, J.-W. Yeh, S.-K. Chen. Competition between elements during mechanical alloying in an octonary multi-principal-element alloy system, J. Alloy. Compd. 481 (2009) 768-775.

[19] A.K. Singh, N. Kumar, A. Dwivedi, A. Subramaniam. A geometrical parameter for the formation of disordered solid solutions in multi-component alloys, Intermet. 53 (2014) 112119.

[20] I.M. Hodge. Enthalpy relaxation and reocvery in amorphous materials, J. Non-Cryst. Solids 169 (1994) 211-266.

[21] S.C. Middleburgh, D.M. King, G.R. Lumpkin, M. Cortie, L. Edwards. Segregation and migration of species in the CrCoFeNi high entropy alloy, J. Alloy. Compd. 599 (2014) 179182.

[22] F.R.d. Boer. Cohesion in metals: transition metal alloys, North-Holland ; Sole distributors for the U.S.A. and Canada, Elsevier Scientific Pub. Co., Amsterdam ; New York, 1988.

[23] D.J.M. King, A.G. McGregor. Alloy Search And Predict (ASAP), http://www.AlloyASAP.com (2015).

[24] A. Benisek, E. Dachs. The vibrational and configurational entropy of disordering in $\mathrm{Cu} 3 \mathrm{Au}$, J. Alloy. Compd. 632 (2015) 585-590.

[25] A. van de Walle, G. Ceder. The effect of lattice vibrations on substitutional alloy thermodynamics, Rev. Mod. Phys. 74 (2002) 11-45.

[26] X. Yang, Y. Zhang. Prediction of high-entropy stabilized solid-solution in multi-component alloys, Mater. Chem. Phys. (2012) 233-238.

[27] S. Middleburgh, R. Grimes, K. Desai, P. Blair, L. Hallstadius, K. Backman, P. Van Uffelen. Swelling due to fission products and additives dissolved within the uranium dioxide lattice, J. Nucl. Mater. (2012) 359-363.

[28] J.W. Wang, Q.T. Guo, O.J. Kleppa. Standard enthalpies of formation of some Th alloys with Group VIII elements (Co, Ni, Ru, Rh, Pd, Ir and Pt), determined by high-temperature direct synthesis calorimetry, J. Alloy. Compd. 313 (2000) 77-84.

[29] H. Yang, W. Tsai, J. Kuo, C. Yang. Solid/liquid interaction between a multicomponent FeCrNiCoMnAl high entropy alloy and molten aluminium, J. Alloy. Compd. (2011) 81768182.

[30] Z.J. Wang, Y.H. Huang, Y. Yang, J.C. Wang, C.T. Liu. Atomic-size effect and solid solubility of multicomponent alloys, Scr. Mater. 94 (2015) 28-31.

[31] G. Kresse, J. Hafner. Ab initio molecular-dynamics for liquid-metals, Phys. Rev. B (1993) 558-561.

[32] G. Kresse, J. Hafner. Ab-inito molecular-dynamics simulation of the liquid-metal amorphous semiconductor transition in germanium, Phys. Rev. B (1994) 14251-14269.

[33] D.J.M. King, S.C. Middleburgh, A.C.Y. Liu, H.A. Tahini, G.R. Lumpkin, M.B. Cortie. Formation and structure of V-Zr amorphous alloy thin films, Acta Mater. 83 (2015) 269275.

[34] D. Pettifor. A quantum-mechanical critique of the Miedema rules for alloy formation, Solid State Phys. 40 (1987) 43-92.

[35] J.H. Zhu, C.T. Liu, L.M. Pike, P.K. Liaw. Enthalpies of formation of binary Laves phases, Intermetallics 10 (2002) 579-595.

[36] J.R. Chelikowsky. Microscopic basis of Miedema's theory of alloy formation, Phys Rev B 25 (1982) 6506-6508. 
[37] Y.L. Zhang, H.S. Liu, Z.P. Jin. Thermodynamic assessment of the Nb-Ti system, Comput. Coupling Phase Diagr. Thermochem. 25 (2001) 305-317.

[38] H. Chou, Y. Chang, S. Chen, J. Yeh. Microstructure, thermophysical and electrical properties in AlxCoCrFeNi $(0<x<2)$ high-entropy alloys, Mater. Sci. Eng. B (2009) 184189.

[39] C. Tong, M. Chen, S. Chen, J. Yeh, T. Shun, S. Lin, S. Chang. Mechanical performance of the AlxCoCrCuFeNi high-entropy alloy system with multiprincipal elements, Metall. Mater. Trans. A (2005) 1264-1271.

[40] S. Praveen, B. Murty, R. Kottada. Alloying behavior in muli-component AlCoCrCuFe and NiCoCrCuFe high entropy alloys, Mater. Sci. Eng. A (2012) 83-89.

[41] Y. Kao, T. Chen, S. Chen, J. Yeh. Microstructure and mechanical property of as-cast, homogenized, and deformed AlxCoCrFeNi $(0<x<2)$ high-entropy alloys, J. Alloy. Compd. (2009) 57-64.

[42] C. Li, M. Zhao, J. Li, Q. Jiang. B2 structure of high-entropy alloys with addition of Al, J. Appl. Phys. (2008) 113504-113501 - 113504-113506.

[43] O.N. Senkov, S.V. Senkova, C. Woodward, D.B. Miracle. Low-density, refractory multiprincipal element alloys of the $\mathrm{Cr}-\mathrm{Nb}-\mathrm{Ti}-\mathrm{V}-\mathrm{Zr}$ system: Microstructure and phase analysis, Acta Mater. 61 (2013) 1545-1557.

[44] S. Lumley, S. Murphy, P. Burr, R. Grimes, P. Chard-Tuckey, M. Wenman. The stability of alloying additions in Zirconium, J. Nucl. Mater. 437 (2013) 122-129.

[45] O.N. Senkov, J.M. Scott, S.V. Senkova, D.B. Miracle, C.F. Woodward. Microstructure and room temperature properties of a high-entropy TaNbHfZrTi alloy, J. Alloy. Compd. 509 (2011) 6043-6048.

[46] Y.D. Wu, Y.H. Cai, T. Wang, J.J. Si, J. Zhu, Y.D. Wang, X.D. Hui. A refractory Hf25Nb25Ti25Zr25 high-entropy alloy with excellent structural stability and tensile properties, Mater. Lett. 130 (2014) 277-280.

[47] Y. Zou, S. Maiti, W. Steurer, R. Spolenak. Size-dependent plasticity in an Nb25Mo25Ta25W25 refractory high-entropy alloy, Acta Mater. 65 (2014) 85-97.

[48] K. Tsai, M. Tsai, J. Yeh. Sluggish diffusion in Co-Cr-Fe-Mn-Ni high-entropy alloys, Acta Mater. (2013) 4887-4897.

[49] S. Abhaya, R. Rajaraman, S. Kalavathi, G. Amarendra. Positron annihilation studies on FeCrCoNi high entropy alloy, J. Alloy. Compd. 620 (2015) 277-282.

[50] C. Ng, S. Guo, J. Luan, S. Shi, C. Liu. Entropy-driven phase stability and slow diffusion kinetics in an Al0.5CoCrCuFeNi high entropy alloy, Intermet. (2012) 165-172.

[51] J.W. Yeh, S.Y. Chang, Y.D. Hong, S.K. Chen, S.J. Lin. Anomalous decrease in X-ray diffraction intensities of $\mathrm{Cu}-\mathrm{Ni}-\mathrm{Al}-\mathrm{Co}-\mathrm{Cr}-\mathrm{Fe}-\mathrm{Si}$ alloy systems with multi-principal elements, Mater. Chem. Phys. 103 (2007) 41-46.

[52] D.J.M. King, S.C. Middleburgh, L. Edwards, G.R. Lumpkin, M. Cortie. (in press) Predicting the crystal structure and phase transitions in high entropy alloys, J. Miner. Metal. Mater. Soc. (2015).

[53] T.T. Zuo, R.B. Li, X.J. Ren, Y. Zhang. Effects of Al and Si addition on the structure and properties of CoFeNi equal atomic ratio alloy, J. Magn. Magn. Mater. 371 (2014) 60-68.

[54] T. Shun, Y. Du. Microstructure and tensile behaviours of FCC A10.3CoCrFeNi high entropy alloy, J. Alloy. Compd. (2009) 157-160.

[55] D. Kim, V. Manga, S. Prins, Z.-K. Liu. First-principles calculations and thermodynamic modeling of the Al-Pt binary system, Calphad 35 (2011) 20-29. 


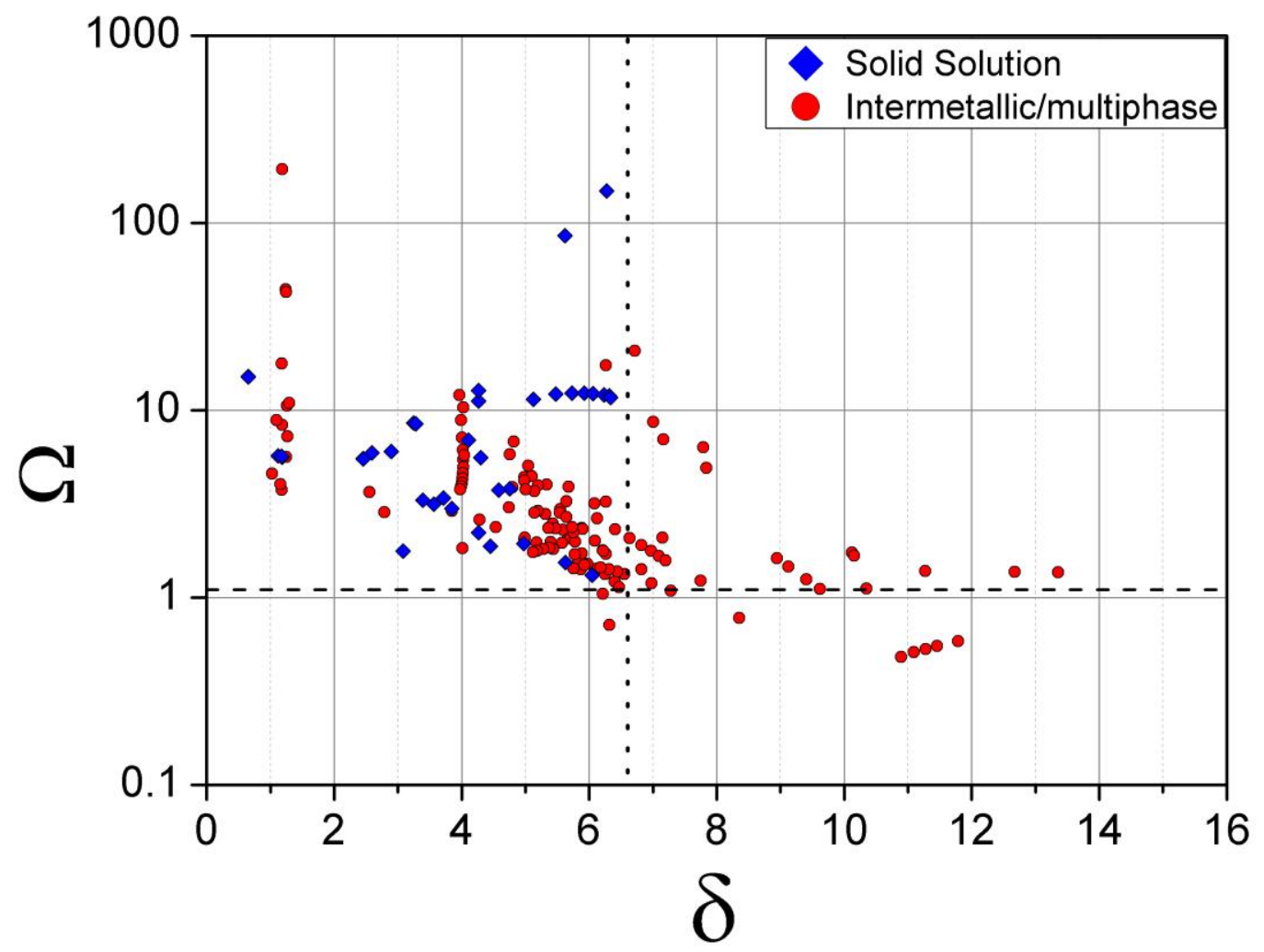

Fig. 1. A comparison of 185 systems, experimentally determined to create a solid solution (blue diamond) or intermetallic/multiphase (red circle) structure, plotted for Yang et al's predictive parameters whereby $\Omega \geq 1.1$ and $\delta \leq 6.6$ predict the formation of a SPHEA. 


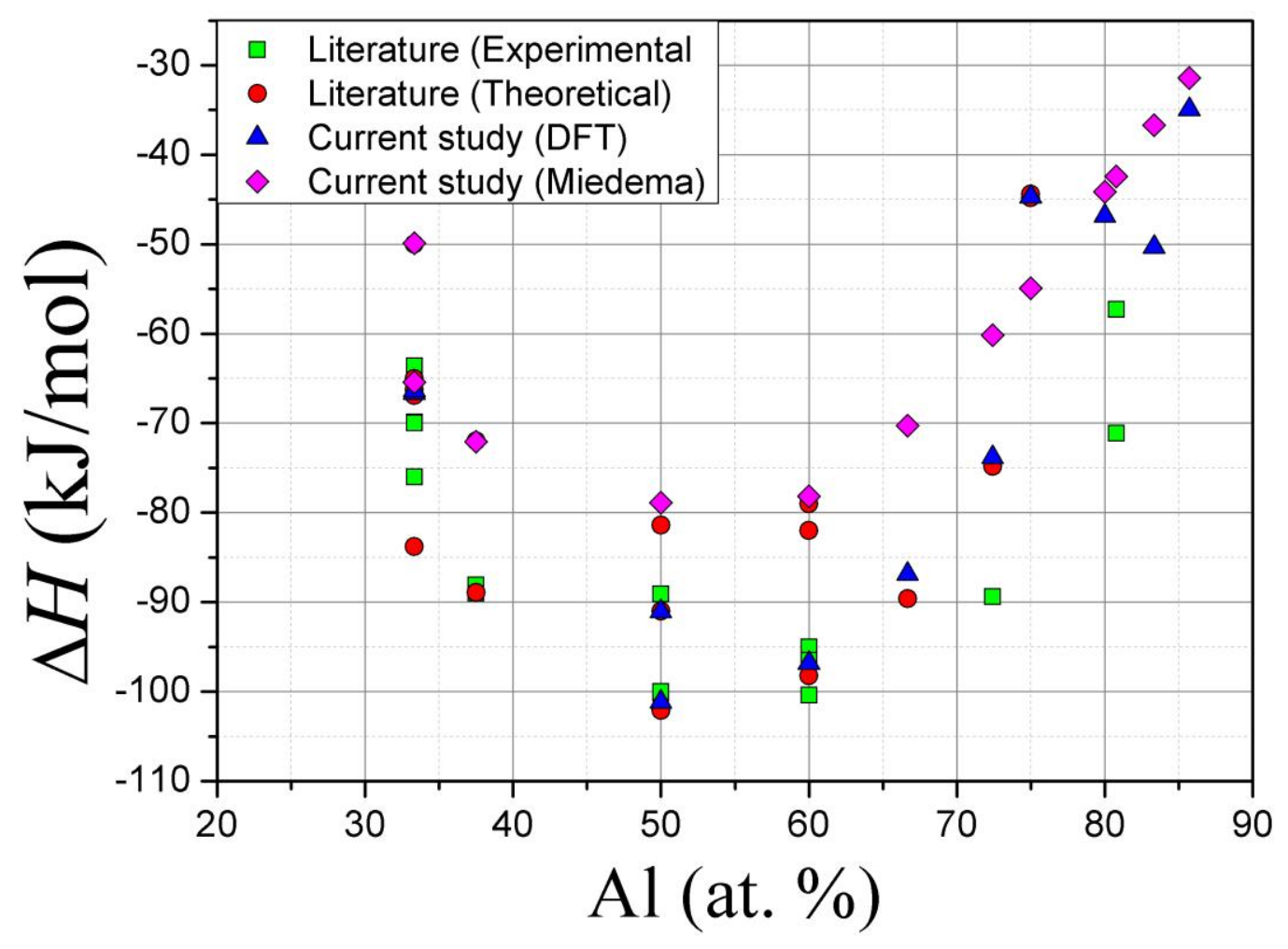

Fig. 2. Formation enthalpies obtained for various compounds of the Pt-Al system, using:

Literature values [57] obtained experimentally (green square) and theoretically (red circle) compared to values obtained from calculations in the current study using DFT (blue triangle) and Miedema's model (magenta diamond). 

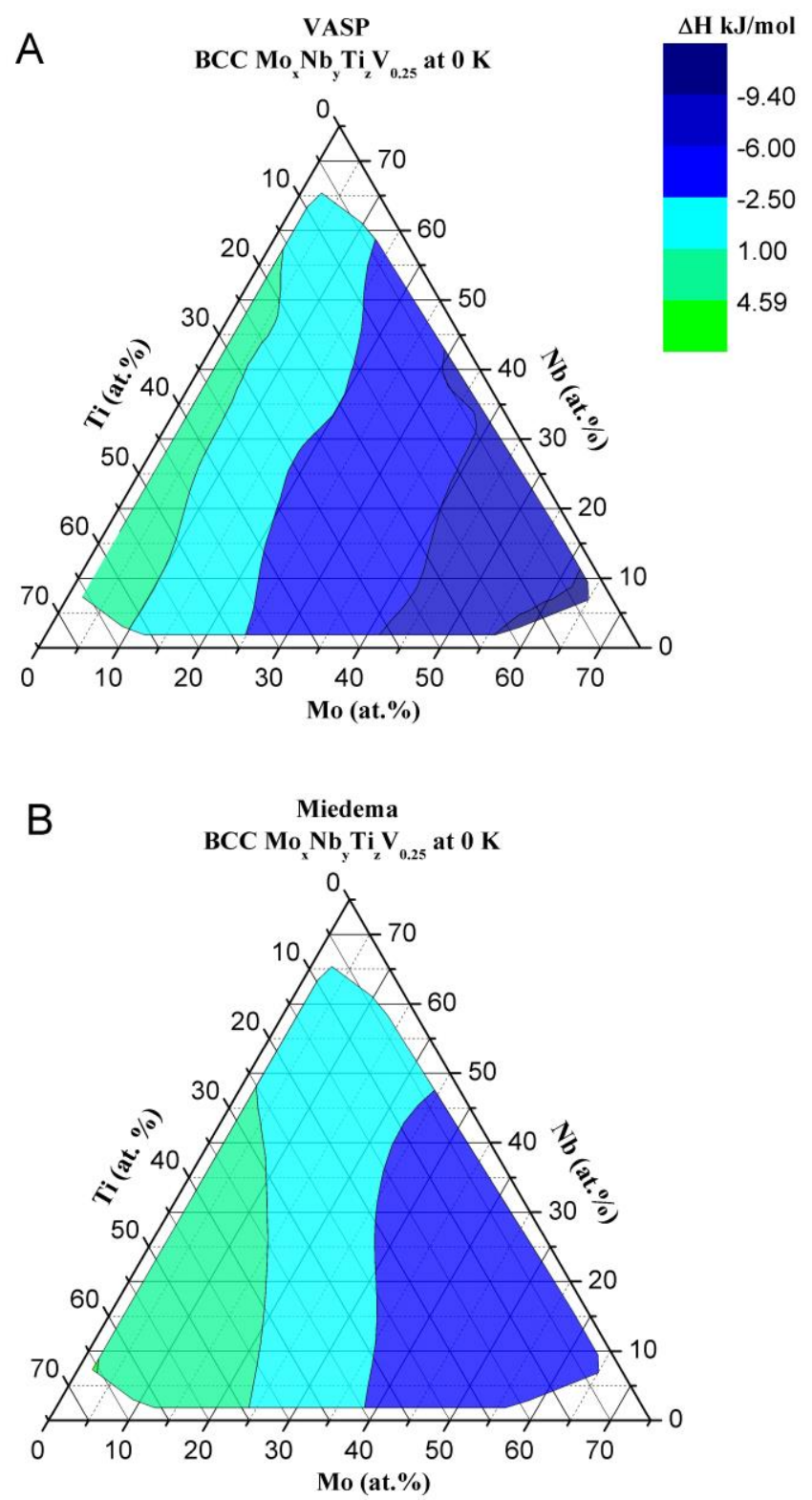

Fig. 3. Formation enthalpies for the formation of a BCC solid solution in the Mo-Nb-Ti- $\mathrm{V}_{0.25}$ system comparing values obtained from A. DFT calculations to B. Miedema's model. The colours ranging from green $\rightarrow$ blue denote the formation enthalpies from $4.59 \rightarrow-$ $9.40 \mathrm{~kJ} / \mathrm{mol}$. 


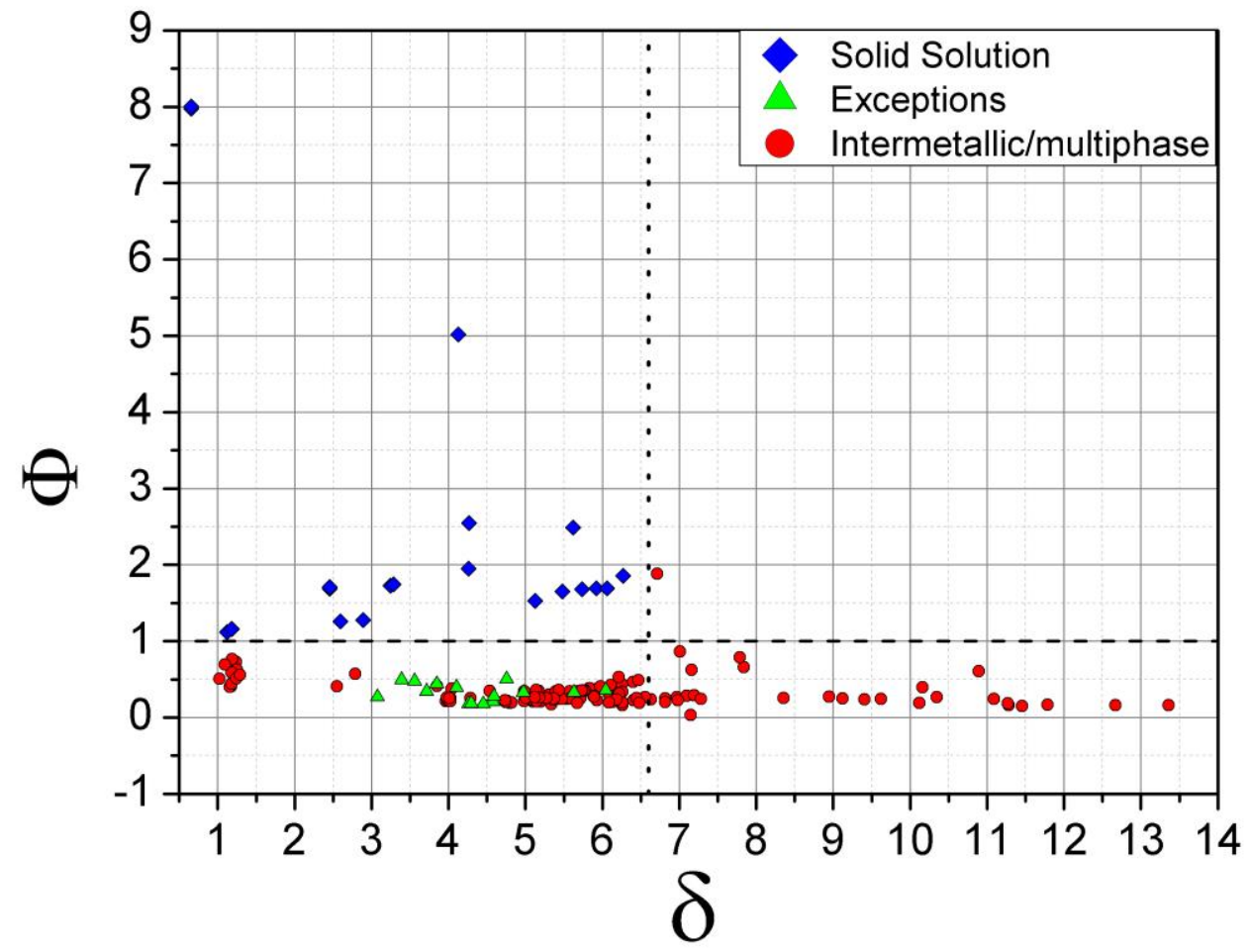

Fig. 4. A comparison of 185 systems experimentally determined to create a solid solution (blue diamond), intermetallic/multiphase (red circle) structure and those that are an exception (green triangle) to the $\Phi \geq 1$ criteria (dashed line) plotted against a geometrical parameter $\delta$. 


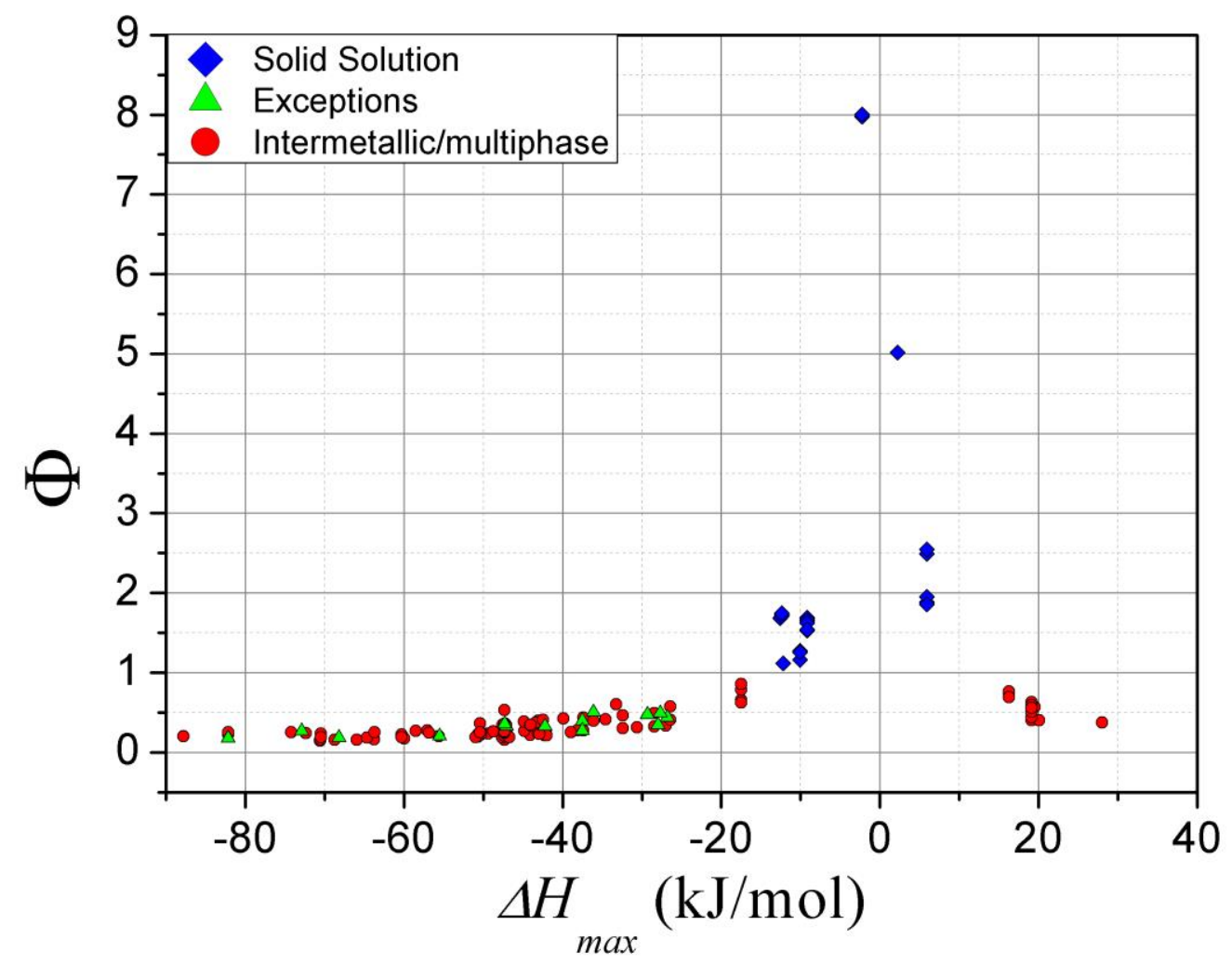

Fig. 5. A comparison of 185 systems experimentally determined to create a solid solution (blue diamond), intermetallic/multiphase (red circle) and those that are an exception to the $\Phi \geq$ 1 criteria (green triangle) plotted against the largest binary enthalpy contribution, $\Delta H_{\max }$. 


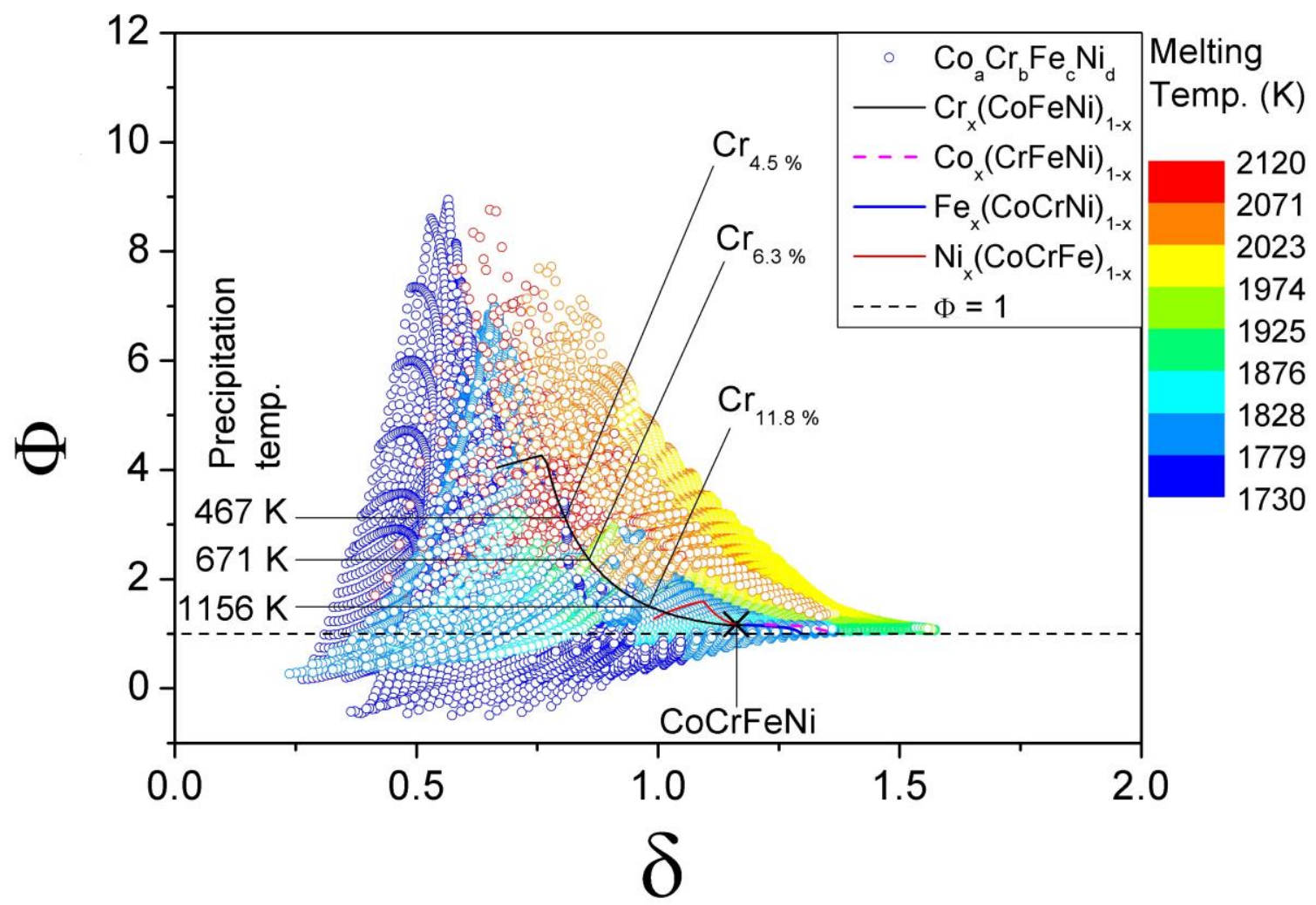

Fig. 6. All possible compositions in the $\mathrm{Co}_{\mathrm{a}} \mathrm{Cr}_{\mathrm{b}} \mathrm{Fe}_{\mathrm{c}} \mathrm{Ni}_{\mathrm{d}}$ system (open coloured circles). Melting temperatures of $1730 \rightarrow 2120 \mathrm{~K}$ are represented as colours from blue $\rightarrow$ red. The equimolar $\mathrm{CoCrFeNi}$ system (cross) and the segregation of each element $\mathrm{Cr}$ (black solid), Co (red solid), Fe (black dashed) and Ni (orange solid) lines are overlayed. The precipitations temperatures at three $\mathrm{Cr}$ contents are displayed and $\Phi=1$ cutoff is denoted by the grey dashed line. 


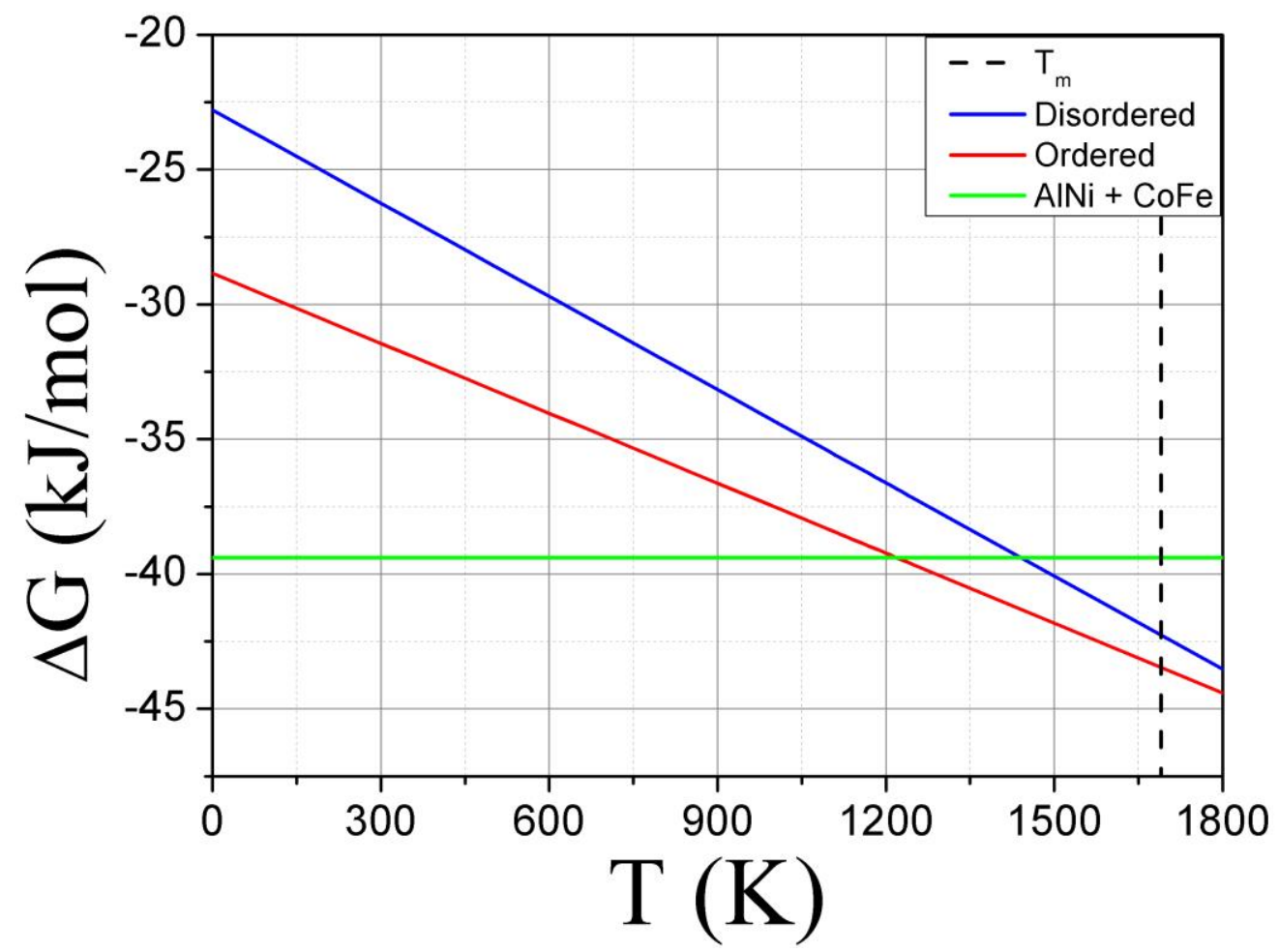

Fig. 7. Gibbs free energy as a function of temperature for competing phases: Disordered solid solution of AlCoFeNi (blue), partially ordered solid solution of $\mathrm{AlCoFeNi}$ (red), and AlNi and CoFe B2 intermetallic compounds (green). The predicted melting temperature is denoted by the black dashed line. 